\title{
A DETERMINISTIC APPROACH FOR OPTIMAL RECURSIVE PREDICTION OF SINGULAR DISCRETE-TIME SYSTEMS
}

\author{
João Y. Ishihara, ${ }^{*}$ Aline F. Bianco, ${ }^{* *}$ \\ Marco H. Terra ${ }^{* *}$ \\ * University of Brasília \\ CEP 70.919-970, Braslia, DF, Brazil. \\ E-mail: ishihara@ene.unb.br \\ ** University of São Paulo at São Carlos \\ CEP 13.566-590, São Carlos, SP, Brazil. \\ E-mail: $\{$ afbianco,terra\}@sel.eesc.usp.br
}

\begin{abstract}
This paper proposes a new representation for the predictor estimates recursion and corresponding Riccati equation for discrete-time, time-variant descriptor systems. The introduced "9-block" form for the predictor and Riccati equation presents an interesting simple and symmetric structure, which enable us to treat directly the most general systems where state and measurement noises are correlated. Copyright (c) 2005 IFAC
\end{abstract}

Keywords: Kalman filtering, predicted estimate, descriptor systems, singular systems, state estimation, discrete-time, Riccati equation.

\section{INTRODUCTION}

In this paper we consider the problem of optimal recursive estimation for discrete-time descriptor systems. The study of estimation and control for such class of systems, also known as singular systems or implicit systems, is motivated by the fact that systems in descriptor formulation frequently arises naturally in economical systems (Luenberger, 1977), image modelling (Hasan and Azim-Sadjani, 1995), and robotics (Mills and Goldenberg, 1989). For discrete-time descriptor systems, the state estimation is presented recursively and the resulting generalized Kalman filter has been intensively studied (see e.g. (Dai, 1989), (Darouach et al., 1993), (Deng and Liu, 1999), (Ishihara et al., July 2004a), (Keller et al., 1992), (Nikoukhah et al., 1999), (Nikoukhah et al., 1992), (Zhang et al., 1999), (Zhang et al., 1996)). Different formulations have been proposed in order to deal with this problem. Each formulation brings some intuitive appeal and opens some possibilities for the study of more general filters. In particular, the Kalman filtering approach addressed as a deterministic optimal trajectory fitting problem has enabled us to propose a robust filter where there exist some uncertain matrices in the system (Ishihara et al., July 2004a), (Ishihara et al., July 2004b). To develop a unified recursive solution for robust filtering problems where there exist uncertainties on all system matrices is more difficult since the filter and Riccati equation expressions for the usual canonical system is already very complex. Besides, in contrast with the usual state space systems, for descriptor system the corresponding deterministic functional for the filtered and predicted approach are different (Ishihara et al., July 2004a). In order to be able to consider directly a very general system where correlation between the state and measurement noises are allowed, we propose here a new ex- 
pression for the filter and corresponding Riccati equation. Usually, the noise correlation is avoided considering an appropriate change of variables. The final expressions for the original system variables although simple to obtain, is in general cumbersome and without any intuitive appeal. For the filtered case, we considered correlated noises between the present output and the present state update in (Bianco et al., 2005). In this paper, we present a very simple and symmetric "9-Block" form of the Kalman estimates recursion and Riccati equation for the predicted estimates. In this new block structure, the predictor estimate representation for descriptor systems with correlated noises between the present state update and the next output is direct. We show that the filter deduced in (Ishihara et al., July 2004a) represents a particular solution for this problem. We hope that the proposed representation can simplify the sensitivity analysis for the Riccati equations and the study of robust filters.

This paper is organized as follows. The deterministic estimation problem is formulated in Section 2. The Kalman filter in predicted form is derived in Section 3. A numerical example is given in Section 4 to demonstrate the applicability of the result.

\section{PROBLEM STATEMENT}

Suppose that there exists a sequence of measurements $z=\left\{z_{0}, z_{1}, \ldots, z_{k}\right\}$ from a certain real dynamical system. We are interested in obtaining a suitable dynamical model which 'explains' the observed measurements and even obtain some knowledge about the values of next measurements, as $z_{k+1}$, for example. An ideal linear dynamical system in its more general form is an implicit or descriptor system

$$
\begin{aligned}
E_{i+1} x_{i+1} & =A_{i} x_{i}, i=0,1,2, \ldots \\
z_{i} & =C_{i} x_{i}
\end{aligned}
$$

where $x_{i}$ is the descriptor variable, or semi-state, which describes the internal behavior of the system; $E_{i+1}, A_{i}$, and $C_{i}$ are real rectangular matrices of appropriated dimensions. With the system matrices fixed, the descriptor Kalman filtering in our context is defined as a deterministic fitting problem (over the entire trajectory). For each state sequence $\left\{x_{0 \mid k}, x_{1 \mid k}, \ldots, x_{k \mid k}, x_{k+1 \mid k}\right\}$ we can define the following fitting errors

$$
\begin{aligned}
{\left[\begin{array}{cc}
B_{w, i} & B_{v, i} \\
D_{w, i} & D_{v, i}
\end{array}\right]\left[\begin{array}{c}
w_{i \mid k} \\
v_{i \mid k}
\end{array}\right] } & :=\left[\begin{array}{c}
E_{i+1} x_{i+1 \mid k}-A_{i} x_{i \mid k} \\
z_{i}-C_{i} x_{i \mid k}
\end{array}\right] \\
i & =0,1, \ldots, k \\
p_{0 \mid k} & :=E_{0} x_{0 \mid k}-A_{-1} \bar{x}_{0}
\end{aligned}
$$

where the matrices $E_{0}$ and $A_{-1}$ are supposed of appropriate dimensions. These matrices can deal with the a priori information on the initial state $x_{0}$, and usually it is supposed $E_{0}=A_{-1}=I$. Now, the deterministic optimal fitting problem is to find a state sequence which minimizes some predefined error functional. Once obtained the minimizing sequence $\left\{\hat{x}_{i \mid k}\right\}$, we can define from (2) the corresponding minimum fitting errors $\hat{w}_{i \mid k}, \hat{v}_{i \mid k}$, $\hat{p}_{0 \mid k}$ so that the complete model which 'explains' the set of measured signals $z=\left\{z_{0}, z_{1}, \ldots, z_{k}\right\}$ turns to be

$$
\begin{aligned}
E_{0} \hat{x}_{0 \mid k} & =A_{-1} \bar{x}_{0}+\hat{p}_{0 \mid k} \\
E_{i+1} \hat{x}_{i+1 \mid k} & =A_{i} \hat{x}_{i \mid k}+B_{w, i} \hat{w}_{i \mid k}+B_{v, i} \hat{v}_{i \mid k} \\
z_{i} & =C_{i} \hat{x}_{i \mid k}+D_{w, i} \hat{w}_{i \mid k}+D_{v, i} \hat{v}_{i \mid k}, \\
i & =0,1, \ldots, k .
\end{aligned}
$$

Our estimation problem is to obtain $\widehat{x}_{k+1 \mid k}$ and $\widehat{x}_{k \mid k}$, i.e., the predicted and filterd estimates. The nomenclature 'filtering' for the estimation problem is justified by the observation that if $C_{i}=I$, $D_{w, i}=0$, and $D_{v, i}=I$ in the model (3)-(5), then we have from (5)

$$
z_{i}=\hat{x}_{i \mid k}+\hat{v}_{i \mid k}
$$

and so, if the signal $\hat{x}_{i \mid k}$ was obtained from the actually measured signal $z_{k}$, the error signal $\hat{v}_{i \mid k}$ has been suppressed from $z_{k}$.

In the next section we will propose one quadratic functional to obtain the predicted estimate recursion. In this functional, the intuitive notion of (relative) degree of uncertainty or, how big we allow each error to be, is dealt with the introduction of positive definite weighting matrices. We will suppose known the positive definite weighting matrices $W_{j}, Z_{i}$, and $P_{0}$ to the errors $w_{j \mid k}, v_{i \mid k}$, and $p_{0 \mid k}$, respectively, for all $i$ and $j$. We suppose that the problem of determining the model parameters is already solved. We follow the widespread procedure in the literature for Kalman filter which postulate that the model is known.

\section{THE KALMAN FILTER IN PREDICTED FORM}

The deterministic predicted least square fitting problem solved in the following is to find a sequence $\left\{\widehat{x}_{0 \mid k}, \ldots, \widehat{x}_{k \mid k}, \widehat{x}_{k+1 \mid k}\right\}$ which minimizes the functional $\mathfrak{F}_{k}\left(\left\{x_{i \mid k}\right\}_{i=0}^{k+1}\right)$

$$
\begin{gathered}
\mathfrak{F}_{k}\left(\left\{x_{i \mid k}\right\}_{i=0}^{k+1}\right):=\frac{1}{2}\left\{\left\|E_{0} x_{0 \mid k}-A_{-1} \bar{x}_{0}\right\|_{P_{0}^{-1}}^{2}\right. \\
\left.+\sum_{i=0}^{k}\left[\begin{array}{c}
w_{i \mid k} \\
v_{i \mid k}
\end{array}\right]^{T}\left[\begin{array}{cc}
W_{i} & X_{i} \\
X_{i}^{T} & Z_{i}
\end{array}\right]^{-1}\left[\begin{array}{c}
w_{i \mid k} \\
v_{i \mid k}
\end{array}\right]\right\}
\end{gathered}
$$


subject to

$$
\begin{aligned}
E_{i+1} x_{i+1 \mid k} & =A_{i} x_{i \mid k}+B_{w, i} w_{i \mid k}+B_{v, i} v_{i \mid k}, \\
z_{i} & =C_{i} x_{i \mid k}+D_{w, i} w_{i \mid k}+D_{v, i} v_{i \mid k}
\end{aligned}
$$

for $k \geq 0$.

For each $k \geq 0$, by rewriting (7) and (8), the original optimization problem is equivalent to the following minimization problem

$$
\min _{\mathbb{X}_{k+1 \mid k}, \mathbb{V}_{k+1 \mid k}}=\frac{1}{2} \mathbb{V}_{k+1 \mid k}^{T} \mathbb{R}_{k}^{-1} \mathbb{V}_{k+1 \mid k}
$$

subject to

$$
\mathbb{Z}_{k}-\mathbb{E}_{k+1} \mathbb{X}_{k+1 \mid k}-\mathbb{K}_{k} \mathbb{V}_{k+1 \mid k}=0
$$

where we adopt the following definitions

$$
\mathbb{V}_{k+1 \mid k}:=\left[\begin{array}{c}
\Theta_{k \mid k} \\
\Theta_{k-1 \mid k} \\
\vdots \\
\Theta_{3 \mid k} \\
\Theta_{2 \mid k} \\
\Theta_{1 \mid k} \\
\Theta_{0 \mid k} \\
\Theta_{-1 \mid k}
\end{array}\right], \mathbb{R}_{k}:=\left[\begin{array}{cccc}
\Omega_{k} & 0 & 0 & 0 \\
0 & \ddots & 0 & 0 \\
0 & 0 & \Omega_{0} & 0 \\
0 & 0 & 0 & \Omega_{-1}
\end{array}\right]
$$

$$
\mathbb{E}_{k+1}:=\left[\begin{array}{ccccccc}
\Sigma_{k+1} & \Delta_{k} & 0 & 0 & 0 & 0 & 0 \\
0 & \Sigma_{k} & \Delta_{k-1} & 0 & 0 & 0 & 0 \\
0 & 0 & \Sigma_{k-1} & \ddots & 0 & 0 & 0 \\
0 & 0 & 0 & \ddots & \Delta_{2} & 0 & 0 \\
0 & 0 & 0 & 0 & \Sigma_{2} & \Delta_{1} & 0 \\
0 & 0 & 0 & 0 & 0 & \Sigma_{1} & \Delta_{0} \\
0 & 0 & 0 & 0 & 0 & 0 & \Sigma_{0}
\end{array}\right]
$$

$$
\mathbb{Z}_{k}:=\left[\begin{array}{c}
\Phi_{k} \\
\Phi_{k-1} \\
\vdots \\
\Phi_{2} \\
\Phi_{1} \\
\Phi_{0} \\
\Phi_{-1}
\end{array}\right], \quad \mathbb{X}_{k+1 \mid k}:=\left[\begin{array}{c}
x_{k+1 \mid k} \\
x_{k \mid k} \\
\vdots \\
x_{2 \mid k} \\
x_{1 \mid k} \\
x_{0 \mid k}
\end{array}\right]
$$

$$
\mathbb{K}_{k}:=\left[\begin{array}{cccccc}
\Psi_{k} & 0 & 0 & 0 & 0 & 0 \\
0 & \Psi_{k-1} & 0 & 0 & 0 & 0 \\
0 & 0 & \ddots & 0 & 0 & 0 \\
0 & 0 & 0 & \Psi_{1} & 0 & 0 \\
0 & 0 & 0 & 0 & \Psi_{0} & 0 \\
0 & 0 & 0 & 0 & 0 & \Psi_{-1}
\end{array}\right]
$$

$$
\Omega_{i}:=\left[\begin{array}{cc}
W_{i} & X_{i} \\
X_{i}^{T} & Z_{i}
\end{array}\right], \quad \Psi_{i}:=\left[\begin{array}{cc}
B_{w, i} & B_{v, i} \\
D_{w, i} & D_{v, i}
\end{array}\right]
$$

$$
\Delta_{i}:=\left[\begin{array}{c}
A_{i} \\
C_{i}
\end{array}\right], \quad \Phi_{i}:=\left[\begin{array}{c}
0 \\
z_{i}
\end{array}\right], \quad \Theta_{i}:=\left[\begin{array}{c}
w_{i \mid k} \\
v_{i \mid k}
\end{array}\right],
$$

for $i \geq 0$

$$
\Sigma_{j}:=\left[\begin{array}{c}
-E_{j} \\
0
\end{array}\right], \text { for } j \geq 1
$$

$$
\Phi_{-1}:=-A_{-1} \bar{x}_{0}, \quad \Omega_{-1}:=P_{0}, \quad \Sigma_{0}:=-E_{0},
$$

$$
\Theta_{-1}:=w_{-1}, \quad \Psi_{-1}:=I
$$

and

$$
w_{-1 \mid 0}:=E_{0} x_{0 \mid 0}-A_{-1} \bar{x}_{0} .
$$

The solvability of above optimization problem is considered in the next lemma.

Lemma 1. For a fixed $k$ suppose that it is given an output sequence $\left\{z_{0}, z_{1}, \ldots, z_{k}\right\}$ and that the following assumptions

A 1. $\left[\begin{array}{cccc}-E_{i+1} & A_{i} & B_{w, i} & B_{v, i} \\ 0 & C_{i} & D_{w, i} & D_{v, i}\end{array}\right]$ has full row rank;

A 2. $E_{i+1}$ has full column rank;

hold for all $0<i \leq k$. Then the data fitting problem (7) - (8) has a unique solution.

Proof: Define an auxiliary variable $w_{-1 \mid 0}$ as in (11). For $k \geq 1$ we have that the Lagrangian function associated to minimization problem (9)(10) is given by

$$
\begin{aligned}
& \mathfrak{L}_{k}:=\frac{1}{2} \mathbb{V}_{k+1 \mid k}^{T} \mathbb{R}_{k}^{-1} \mathbb{V}_{k+1 \mid k}+ \\
& \Lambda_{k \mid k}^{T}\left(\mathbb{Z}_{k}-\mathbb{E}_{k+1} \mathbb{X}_{k+1 \mid k}-\mathbb{K}_{k} \mathbb{V}_{k \mid k}\right)
\end{aligned}
$$

where the Lagrange multiplier is

$$
\begin{aligned}
\Lambda_{k \mid k} & :=\left[\begin{array}{c}
\mathcal{L}_{k \mid k} \\
\mathcal{L}_{k-1 \mid k} \\
\vdots \\
\mathcal{L}_{0 \mid k} \\
\mathcal{L}_{-1 \mid k}
\end{array}\right], \quad \mathcal{L}_{j \mid k}:=\left[\begin{array}{c}
\mu_{j \mid k} \\
\lambda_{j \mid k}
\end{array}\right], \quad 0 \leq j \leq k, \\
\mathcal{L}_{-1 \mid k}: & =\mu_{-1 \mid k} .
\end{aligned}
$$




$$
\widehat{x}_{k+1 \mid k}:=\left[\begin{array}{l}
0 \\
0 \\
0 \\
0 \\
0 \\
0 \\
0 \\
0 \\
I
\end{array}\right]^{T}\left[\begin{array}{ccccccccc}
P_{k \mid k-1} & 0 & 0 & 0 & 0 & I & 0 & 0 & 0 \\
0 & W_{k} & X_{k} & 0 & 0 & 0 & I & 0 & 0 \\
0 & X_{k}^{T} & Z_{k} & 0 & 0 & 0 & 0 & I & 0 \\
0 & 0 & 0 & 0 & 0 & A_{k} & B_{w, k} & B_{v, k} & -E_{k+1} \\
0 & 0 & 0 & 0 & 0 & C_{k} & D_{w, k} & D_{v, k} & 0 \\
I & 0 & 0 & A_{k}^{T} & C_{k}^{T} & 0 & 0 & 0 & 0 \\
0 & I & 0 & B_{w, k}^{T} & D_{w, k}^{T} & 0 & 0 & 0 & 0 \\
0 & 0 & I & B_{v, k}^{T} & D_{v, k}^{T} & 0 & 0 & 0 & 0 \\
0 & 0 & 0 & -E_{k+1}^{T} & 0 & 0 & 0 & 0 & 0
\end{array}\right]^{-1}\left[\begin{array}{c}
\widehat{x}_{k \mid k-1} \\
0 \\
0 \\
0 \\
z_{k} \\
0 \\
0 \\
0 \\
0
\end{array}\right]
$$

$$
P_{k+1 \mid k}:=-\left[\begin{array}{l}
0 \\
0 \\
0 \\
0 \\
0 \\
0 \\
0 \\
0 \\
I
\end{array}\right]^{T}\left[\begin{array}{ccccccccc}
P_{k \mid k-1} & 0 & 0 & 0 & 0 & I & 0 & 0 & 0 \\
0 & W_{k} & X_{k} & 0 & 0 & 0 & I & 0 & 0 \\
0 & X_{k}^{T} & Z_{k} & 0 & 0 & 0 & 0 & I & 0 \\
0 & 0 & 0 & 0 & 0 & A_{k} & B_{w, k} & B_{v, k} & -E_{k+1} \\
0 & 0 & 0 & 0 & 0 & C_{k} & D_{w, k} & D_{v, k} & 0 \\
I & 0 & 0 & A_{k}^{T} & C_{k}^{T} & 0 & 0 & 0 & 0 \\
0 & I & 0 & B_{w, k}^{T} & D_{w, k}^{T} & 0 & 0 & 0 & 0 \\
0 & 0 & I & B_{v, k}^{T} & D_{v, k}^{T} & 0 & 0 & 0 & 0 \\
0 & 0 & 0 & -E_{k+1}^{T} & 0 & 0 & 0 & 0 & 0
\end{array}\right]^{-1}\left[\begin{array}{l}
0 \\
0 \\
0 \\
0 \\
0 \\
0 \\
0 \\
0 \\
I
\end{array}\right]
$$

Setting the differentiates of $\mathfrak{L}_{k}$ to zero and introducing the auxiliary variable $\Upsilon_{k \mid k}:=-\mathbb{R}_{k}^{-1} \mathbb{V}_{k+1 \mid k}$ we have that

$$
\left(\Lambda_{k \mid k}, \mathbb{V}_{k+1 \mid k}, \mathbb{X}_{k+1 \mid k}\right)=\left(\widehat{\Lambda}_{k \mid k}, \widehat{\mathbb{V}}_{k+1 \mid k}, \widehat{\mathbb{X}}_{k+1 \mid k}\right)
$$

is a minimum point of $\mathfrak{J}_{k}$ only if

$$
\begin{array}{r}
\left(\Upsilon_{k \mid k}, \Lambda_{k \mid k}, \mathbb{V}_{k+1 \mid k}, \mathbb{X}_{k+1 \mid k}\right)= \\
\quad\left(\widehat{\Upsilon}_{k \mid k}, \widehat{\Lambda}_{k \mid k}, \widehat{\mathbb{V}}_{k+1 \mid k}, \widehat{\mathbb{X}}_{k+1 \mid k}\right)
\end{array}
$$

is a solution of the system

$$
\begin{aligned}
\mathbb{R}_{k} \Upsilon_{k \mid k}+\mathbb{V}_{k+1 \mid k} & =0 \\
\mathbb{K}_{k} \mathbb{V}_{k+1 \mid k}+\mathbb{E}_{k+1} \mathbb{X}_{k+1 \mid k} & =\mathbb{Z}_{k} \\
\Upsilon_{k \mid k}+\mathbb{K}_{k}^{T} \Lambda_{k \mid k} & =0 \\
\mathbb{E}_{k+1}^{T} \Lambda_{k} & =0
\end{aligned}
$$

or equivalently,

$$
\left[\begin{array}{cccc}
\mathbb{R}_{k} & 0 & I & 0 \\
0 & 0 & \mathbb{K}_{k} & \mathbb{E}_{k+1} \\
I & \mathbb{K}_{k}^{T} & 0 & 0 \\
0 & \mathbb{E}_{k+1}^{T} & 0 & 0
\end{array}\right]\left[\begin{array}{c}
\Upsilon_{k \mid k} \\
\Lambda_{k \mid k} \\
\mathbb{V}_{k+1 \mid k} \\
\mathbb{X}_{k+1 \mid k}
\end{array}\right]=\left[\begin{array}{c}
0 \\
\mathbb{Z}_{k} \\
0 \\
0
\end{array}\right]
$$

Writing in terms of original parameters, it is easy to check that if the assumptions $A 1-A 2$ hold then the leftmost matrix in (15) is invertible. This assure that the solution is unique.

Theorem 3.1. Suppose that assumptions $A 1-A 2$ hold and it is given a sequence $\left\{z_{0}, z_{1}, \ldots\right\}$. Then the successive optimal estimates $\hat{x}_{k+1 \mid k}$ resulting from the solution of the data fitting problem (7) - (8) can alternatively be obtained from the following recursive algorithm
Step 0 (Initial Conditions)

$$
\begin{aligned}
& P_{0 \mid-1}:=P_{0} \\
& \widehat{x}_{0 \mid-1}:=\bar{x}_{0} .
\end{aligned}
$$

Step $k$ : Update $\left\{\hat{x}_{k \mid k-1}, P_{k \mid k-1}\right\}$ to $\left\{\hat{x}_{k+1 \mid k}, P_{k+1 \mid k}\right\}$ as in (12) and (13).

Observe that although we have considered a more general linear system than the usual in the literature, the final expressions (12) and (13) have a central matrix with symmetric structure which is very simple. The results were obtained directly without the need of rewriting the original problem in an augmented system.

In order to compare Theorem 3.1 with the results reported in (Ishihara et al., July 2004a), considers $B_{v, k}=0, D_{w, k}=0, D_{v, k}=I, X_{k}=0$. If in addition $\left[A_{k} B_{w, k}\right]$ has full row rank, then we can rewrite (12) and (13) as

$$
\begin{aligned}
& P_{k+1 \mid k}:=-\left[\begin{array}{lll}
0 & 0 & I
\end{array}\right] \\
& {\left[\begin{array}{ccc}
Y & -A_{k} P_{k \mid k-1} C_{k}^{T} & E_{k+1} \\
-C_{k} P_{k \mid k-1} A_{k}^{T} & Z_{k}+C_{k} P_{k \mid k-1} C_{k}^{T} & 0 \\
E_{k+1}^{T} & 0 & 0
\end{array}\right]^{-1}\left[\begin{array}{l}
0 \\
0 \\
I
\end{array}\right]}
\end{aligned}
$$

and 


$$
\widehat{x}_{k \mid k}=\left[\begin{array}{c}
0 \\
0 \\
0 \\
0 \\
0 \\
I \\
0 \\
0 \\
0
\end{array}\right]^{T}\left[\begin{array}{ccccccccc}
P_{k \mid k-1} & 0 & 0 & 0 & 0 & I & 0 & 0 & 0 \\
0 & W_{k} & X_{k} & 0 & 0 & 0 & I & 0 & 0 \\
0 & X_{k}^{T} & Z_{k} & 0 & 0 & 0 & 0 & I & 0 \\
0 & 0 & 0 & 0 & 0 & A_{k} & B_{w, k} & B_{v, k} & -E_{k+1} \\
0 & 0 & 0 & 0 & 0 & C_{k} & D_{w, k} & D_{v, k} & 0 \\
I & 0 & 0 & A_{k}^{T} & C_{k}^{T} & 0 & 0 & 0 & 0 \\
0 & I & 0 & B_{w, k}^{T} & D_{w, k}^{T} & 0 & 0 & 0 & 0 \\
0 & 0 & I & B_{v, k}^{T} & D_{v, k}^{T} & 0 & 0 & 0 & 0 \\
0 & 0 & 0 & -E_{k+1}^{T} & 0 & 0 & 0 & 0 & 0
\end{array}\right]^{-1}\left[\begin{array}{c}
\widehat{x}_{k \mid k-1} \\
0 \\
0 \\
0 \\
z_{k} \\
0 \\
0 \\
0 \\
0
\end{array}\right]
$$

$$
\begin{aligned}
& \widehat{x}_{k+1 \mid k}:=\left[\begin{array}{lll}
0 & 0 & I
\end{array}\right] \\
& {\left[\begin{array}{ccc}
Y & -A_{k} P_{k \mid k-1} C_{k}^{T} & E_{k+1} \\
-C_{k} P_{k \mid k-1} A_{k}^{T} & Z_{k}+C_{k} P_{k \mid k-1} C_{k}^{T} & 0 \\
E_{k+1}^{T} & 0 & 0
\end{array}\right]^{-1} \times} \\
& \text { or } \left.\begin{array}{c}
A_{k} \widehat{x}_{k \mid k-1} \\
z_{k}-C_{k} \widehat{x}_{k \mid k-1} \\
0
\end{array}\right] \\
& P_{k+1 \mid k}:=\left(E _ { k + 1 } ^ { T } \left(Y-A_{k} P_{k \mid k-1} C_{k}^{T} \times\right.\right. \\
& \left.\left.\left(Z_{k}+C_{k} P_{k \mid k-1} C^{T}\right)^{-1} C_{k} P_{k \mid k-1} A_{k}^{T}\right)^{-1} E_{k+1}\right)^{-1}
\end{aligned}
$$

or

and

$$
\begin{aligned}
& \widehat{x}_{k+1 \mid k}=P_{k+1 \mid k} E_{k+1}^{T}\left(Y-A_{k} P_{k \mid k-1} C_{k}^{T} \times\right. \\
& \left.\left(Z_{k}+C_{k} P_{k \mid k-1} C^{T}\right)^{-1} C_{k} P_{k \mid k-1} A_{k}^{T}\right)^{-1} \times \\
& A_{k} \widehat{x}_{k \mid k-1}+P_{k+1 \mid k} E_{k+1}^{T}\left(Y-A_{k} P_{k \mid k-1} C_{k}^{T} \times\right. \\
& \left.\left(Z_{k}+C_{k} P_{k \mid k-1} C_{k}^{T}\right)^{-1} C_{k} P_{k \mid k-1} A_{k}^{T}\right)^{-1} \times \\
& A_{k} P_{k \mid k-1} C_{k}^{T}\left(Z_{k}+C_{k} P_{k \mid k-1} C_{k}^{T}\right)^{-1} \times \\
& \left(z_{k}-C_{k} \widehat{x}_{k \mid k-1}\right)
\end{aligned}
$$

where $Y:=A_{k} P_{k \mid k-1} A_{k}^{T}+B_{w, k} W_{k} B_{w, k}^{T}$.

For the usual state space $\left(E_{k}=I\right)$, the algorithm in Theorem 3.1 collapses to the usual Kalman filter in predictor form, which confirms that the deterministic fit estimate is equal to the Kalman filter estimate obtained from stochastic reasonings.

From the recursive solution obtained in Theorem 3.1 , we can also re-state $(7)-(8)$ considering the optimization problem of the next lemma.

Lemma 2. The optimal predicted estimates algorithm of Theorem 3.1 can be obtained by the solution of (19)

$$
\begin{aligned}
& \left(\widehat{x}_{k+1 \mid k}, \widehat{x}_{k \mid k}\right):=\min _{x_{k}, x_{k+1}} \\
& \left\{\frac{1}{2}\left[\begin{array}{c}
x_{k}-\widehat{x}_{k \mid k-1} \\
w_{k} \\
v_{k}
\end{array}\right]^{T}\left[\begin{array}{ccc}
P_{k \mid k-1} & 0 & 0 \\
0 & W_{k} & X_{k} \\
0 & X_{k}^{T} & Z_{k}
\end{array}\right]^{-1}[\bullet\}\right.
\end{aligned}
$$

From solution of (19), the filtered estimate $\hat{x}_{k \mid k}$ can be obtained in measurement update form (17). Note that even for the usual state space systems, where $E_{i}=I$, the introduction of noise cross-correlation leads to an usual expression which is more complex than (17). As in (Bianco et al., 2005) the filtered estimate $\hat{x}_{k \mid k}$ recursion is obtained, one could expect that the time-andmeasurement-update form of Kalman filter could be obtained. However, we observe that the funcional in (7) and the corresponding functional used in (Bianco et al., 2005) are different, and consequently the obtained estimations are the same only under some especial conditions (for the usual state space system where $E_{i}=I$, both functionals furnish the same estimates).

\section{NUMERICAL EXAMPLE}

This example considers the descriptor system, with correlated noises, described by (8), where the system matrices are given by

$$
\begin{gathered}
E_{i+1}=E=\left[\begin{array}{cc}
1 & 0 \\
0 & 1 \\
2 & 0.7
\end{array}\right] ; \quad A_{i}=A=\left[\begin{array}{cc}
0.3 & 0 \\
0 & 0.2 \\
0.34 & 0.21
\end{array}\right] ; \\
B_{w, i}=B_{w}=\left[\begin{array}{cc}
0.4 & 0.1 \\
0.1 & 6 \\
0.87 & 0.62
\end{array}\right] ; \quad B_{v, i+1}=B_{v}=\left[\begin{array}{c}
1 \\
1 \\
2.7
\end{array}\right] ; \\
C_{i}=C=\left[\begin{array}{cc}
1.4 & 0.8
\end{array}\right] ; \quad D_{w, i}=D_{w}=\left[\begin{array}{ll}
1.4 & 1.4
\end{array}\right] ; \\
D_{v, i+1}=D_{v}=1
\end{gathered}
$$

and the covariance of $w_{k}$ and $v_{k}$ (with the crossterm) are given respectively by

$$
W=\left[\begin{array}{cc}
7 & 0.0002 \\
0.0002 & 0.001
\end{array}\right] ; \quad Z=0.5 ; \quad X=\left[\begin{array}{c}
0.01 \\
0.0001
\end{array}\right]
$$

The simulation results based on the filter developed in Theorem 3.1, are presented in Figures 1 and 2 .

subject to $(8)$. 


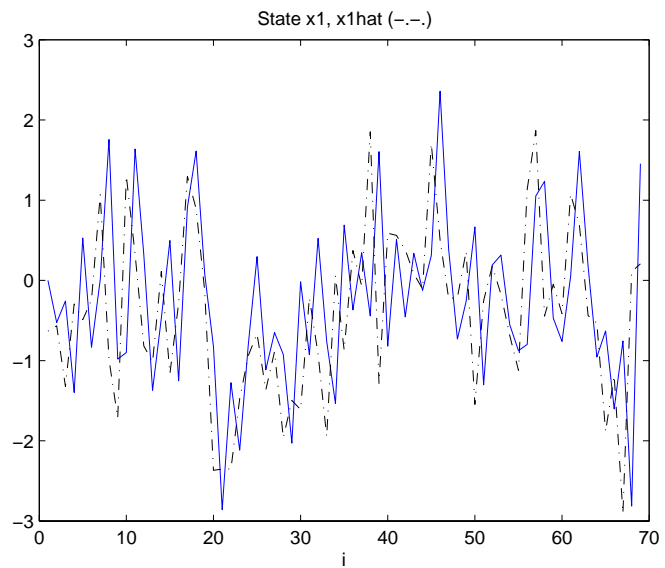

Figure 1. True value of state $\mathrm{x} 1(\mathrm{k})$ and predicted estimate.

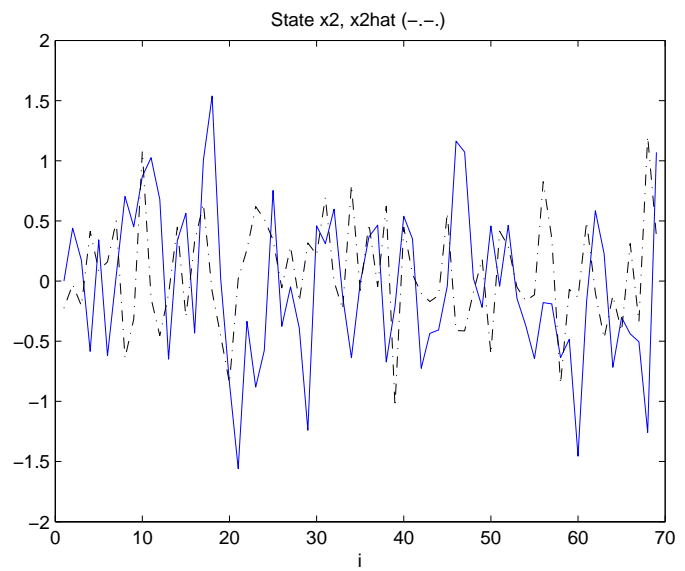

Figure 2. True value of state $\mathrm{x} 2(\mathrm{k})$ and predicted estimate.

\section{CONCLUSION}

We have considered in this paper a general formulation of the Kalman prediction recursion for descriptor systems where all system parameters are present. We introduced a "9-block" form for the filter and Riccati equation which present an interesting simple and symmetric structure. This simple estructure opens the possibility of simplify the Riccati equation sensitivity analysis and of solving the robust recursive filtering problem in which uncertainties are present in all parameter matrices. These problems will be considered in a future work.

\section{Acknowledgments}

This work is supported by FAPESP (Fundação de Amparo à Pesquisa do Estado de São Paulo Brazil) under grants 03/12574-0 and 04/03826-8 and Fundação de Empreendimentos Científicos e Tecnológicos (FINATEC).

\section{REFERENCES}

Bianco, A. F., J. Y. Ishihara and M. H. Terra (2005). A deterministic approach for general discrete-time kalman filter for singular systems. Proceedings of the 24th American Control Conference, Portland, Oregon, USA.

Dai, L. (1989). Impulsive modes and causality in singular systems. Int. J. Control 50(4), 12671281.

Darouach, M., M. Zasadzinski and D. Mehdi (1993). State estimation of stochastic singular linear systems. Int. J. Syst. Sci. 24(2), 345354.

Deng, Z. and Y. Liu (1999). Descriptor Kalman estimators. Int. J. Syst. Sci. 30(11), 12051212.

Hasan, M. A. and M. R. Azim-Sadjani (1995). Noncausal image modeling using descriptor approach. IEEE Trans. Circuits Syst. II 42(8), 536-540.

Ishihara, J. Y., M. H. Terra and J. C. T. Campos (July 2004a). Kalman estimators for discretetime descriptor systems. Proceedings of the 2004 American Control Conference, Boston, Massachusetts pp. 188-193.

Ishihara, J. Y., M. H. Terra and J. C. T. Campos (July 2004b). Robust Kalman filter for descriptor systems. Proceedings of the 2004 American Control Conference, Boston, Massachusetts pp. 194-199.

Keller, J. Y., S. Nowakowski and M. Darouach (1992). State estimation and failure detection in singular systems. Control-Theory and $A d$ vanced Technology 8(4), 755-762.

Luenberger, D. V. (1977). Dynamic equations in descriptor form. IEEE Trans. Automat. Contr. 22(3), 312-321.

Mills, J. K. and A. A. Goldenberg (1989). Force and position control of manipulators during constrained motion tasks. IEEE Trans. Robot. Automat. 68, 30-46.

Nikoukhah, R., A. L. Willsky and B. C. Levy (1992). Kalman filtering and Riccati equations for decriptor systems. IEEE Trans. Automat. Contr. 37(9), 1325-1342.

Nikoukhah, R., S. L. Campbell and F. Delebecque (1999). Kalman filtering for general discretetime linear systems. IEEE Trans. Automat. Contr. 44(10), 1829-1839.

Zhang, H. S., L. Xie and Y. C. Soh (1999). Optimal recurisve filtering, predition and smoothing for singular stochastic discretetime systems. IEEE Trans. Automat. Contr. 44(11), 2154-2158.

Zhang, H., T. Chai and X. Liu (1996). A unified approach to optimal estimation for stochastic singular system. 13th Trienal World Congress, San Francisco, USA pp. 487-492. 